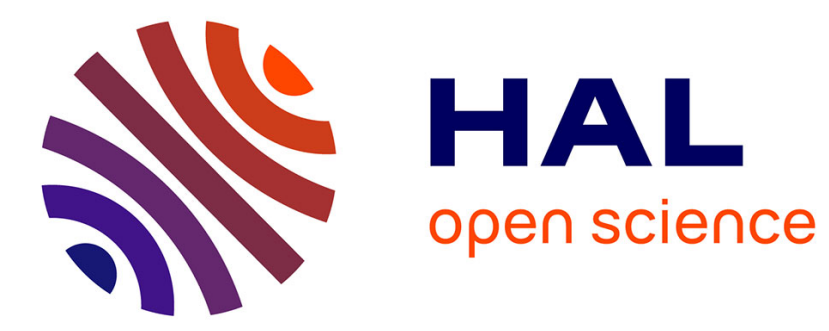

\title{
L'entrée en vigueur du Protocole de Kyoto le 16 février 2005 : quelles implications pour les forêts françaises?
}

Alain Chaudron, Michel-Paul Morel, Valérie Merckx, Guillaume Gaborit

\section{To cite this version:}

Alain Chaudron, Michel-Paul Morel, Valérie Merckx, Guillaume Gaborit. L'entrée en vigueur du Protocole de Kyoto le 16 février 2005 : quelles implications pour les forêts françaises?. Revue forestière française, 2005, 57 (6), pp.479-495. 10.4267/2042/5012 . hal-03449444

\section{HAL Id: hal-03449444 \\ https://hal.science/hal-03449444}

Submitted on 25 Nov 2021

HAL is a multi-disciplinary open access archive for the deposit and dissemination of scientific research documents, whether they are published or not. The documents may come from teaching and research institutions in France or abroad, or from public or private research centers.
L'archive ouverte pluridisciplinaire HAL, est destinée au dépôt et à la diffusion de documents scientifiques de niveau recherche, publiés ou non, émanant des établissements d'enseignement et de recherche français ou étrangers, des laboratoires publics ou privés. 


\title{
POLITIOUES ET INSTITUTIONS
}

\section{L'entrée en Vigueur du Protocole de Kyoto LE 16 FÉVRIER 2005 : QUELLES IMPLICATIONS POUR LES FORÊTS FRANÇAISES?}

\author{
Alain Chaudron - Michel-Paul Morel - Valérie MerckX - Guillaume Gaborit
}

Avertissement

Cet article a été envoyé à la publication en septembre 2005 et contient donc des informations à jour à cette date. Mais le sujet est en perpétuelle évolution et nécessiterait une actualisation permanente... Pour ne citer que deux exemples, la première Conférence des Parties agissant comme réunion des Parties au Protocole de Kyoto (COP-MOP1) s'est bien réunie du 28 novembre au 9 décembre 2005 à Montréal et a avalisé les accords de Marrakech ; d'autre part, la France a bien envoyé à la Commission européenne son rapport sur les quantités attribuées en janvier 2006, indiquant notamment qu'elle ne retenait que l'activité "gestion forestière" au titre de l'article 3.4 pour la première période d'engagement du Protocole de Kyoto.

Les auteurs prient les lecteurs de les excuser de cet inconvénient.

On connaît depuis environ deux siècles la capacité des forêts à fixer du carbone. Mais ce n'est que très récemment, à la faveur des processus internationaux engagés pour limiter les émissions d'origine anthropique de gaz à effet de serre, qu'est apparu l'intérêt de comptabiliser les flux et stocks de carbone engendrés par la forêt. Les contraintes des négociations internationales font que les règles applicables à une telle comptabilité sont caractérisées notamment par une certaine complexité, la nécessité de conventions comptables et un réalisme limité par la disponibilité de données ou de méthodes, voire par le jeu des forces géopolitiques. Après l'entrée en vigueur récente du Protocole de Kyoto, il est temps de faire le point sur le processus en cours et ses conséquences pour les forêts françaises. Cet article présente successivement la Convention-cadre sur les changements climatiques et le Protocole de Kyoto qui en découle, les textes d'application pour leurs parties concernant spécifiquement le secteur forestier, les modalités d'application pour la forêt française, enfin les perspectives futures d'un processus qui, non seulement a régulièrement évolué lors de réunions annuelles, mais encore est appelé à poursuivre son développement.

\section{LA CONVENTION-CADRE DES NATIONS UNIES SUR LES CHANGEMENTS CLIMATIQUES ET LE PROTOCOLE DE KYOTO}

\section{La Convention-cadre des Nations Unies sur les changements climatiques (CCNUCC)}

L'assemblée générale des Nations Unies a mis en place en 1990 un comité intergouvernemental de négociation chargé d'élaborer une convention-cadre sur les changements climatiques. Le 
projet de convention a été adopté en mai 1992 à New York et la convention a été ouverte à la signature lors du Sommet de la Terre à Rio de Janeiro en juin 1992. Elle est entrée en vigueur le 21 mars 1994 ; elle a été ratifiée par 189 pays, dont les États-Unis. La France l'a ratifiée et publiée au Journal Officiel (loi n 94-106 du 5 février 1994 et décret nº 94-501 du 20 juin 1994). Elle s'applique en métropole et dans toutes les collectivités d'Outre-Mer.

Son objectif ultime est de "stabiliser (...) les concentrations de gaz à effet de serre dans l'atmosphère à un niveau qui empêche toute perturbation anthropique dangereuse du système climatique ». Mais il n'y a pas d'objectifs chiffrés, ni de dispositif visant au respect des engagements.

La convention donne notamment une définition des "réservoirs", des "puits" et des "sources" : voir encadré 1 (ci-dessous).

\section{ENCADRÉ 1 \\ DÉFINITIONS
FIGURANT À L’ARTICLE PREMIER DE LA CONVENTION-CADRE}

On entend par "gaz à effet de serre" les constituants gazeux de l'atmosphère, tant naturels qu'anthropiques, qui absorbent et ré-émettent le rayonnement infrarouge.

On entend par "réservoir" un ou plusieurs constituants du système climatique qui retiennent un gaz à effet de serre ou un précurseur de gaz à effet de serre.

On entend par "puits" tout processus, toute activité ou tout mécanisme, naturel ou artificiel, qui élimine de l'atmosphère un gaz à effet de serre, un aérosol ou un précurseur de gaz à effet de serre.

On entend par "source" tout processus ou activité qui libère dans l'atmosphère un gaz à effet de serre, un aérosol ou un précurseur de gaz à effet de serre.

Les États industrialisés ou à économies en transition, Parties à la Convention, sont listés à l'annexe I de la Convention. Ils doivent communiquer chaque année au Secrétariat de la Convention l'inventaire de leurs émissions anthropiques par les sources et de leurs absorptions par les puits de gaz à effet de serre.

\section{Le Protocole de Kyoto}

Dès sa première Conférence des Parties, à Berlin en 1995, la Convention a adopté le mandat de négociation d'un instrument juridique contraignant devant la compléter : le Protocole de Kyoto a été adopté lors de la troisième Conférence des Parties, en 1997.

Ses modalités d'application ont été adoptées à Marrakech, en novembre 2001, lors de la septième Conférence des Parties ( $\mathrm{COP}_{7}$ ), ce qui a ouvert la voie à sa ratification.

Son article 25.1 définit ses conditions d'entrée en vigueur (règle des "deux fois cinquante-cinq") : "Le présent Protocole entre en vigueur le quatre-vingt-dixième jour qui suit la date du dépôt de leurs instruments de ratification, d'acceptation, d'approbation ou d'adhésion par 55 Parties à la Convention au minimum, parmi lesquelles les Parties visées à l'annexe I dont les émissions totales de dioxyde de carbone représentaient en 1990 au moins 55 \% du volume total des émissions de dioxyde de carbone de l'ensemble des Parties visées à cette annexe ". 
Lorsque les États-Unis (34\% des émissions des pays de l'annexe I) ont annoncé qu'ils ne le ratifieraient pas, la ratification russe (17\%) est devenue indispensable.

Finalement, après sa ratification par la Fédération de Russie en novembre 2004, le Protocole de Kyoto est entré en vigueur le 16 février 2005. À ce jour, il a été ratifié par 156 pays, à l'exception notable des États-Unis et de l'Australie, et par la Communauté européenne. La France l'a ratifié et publié au Journal officiel (loi $n^{\circ}$ 2000-645 du 10 juillet 2000 et décret $n^{\circ}$ 2005-295 du 22 mars 2005). Contrairement à la convention-cadre, il s'applique en métropole et dans les seuls départements/régions d’Outre-Mer (les “régions ultra-périphériques” européennes).

En application du Protocole, les pays développés et en transition (Parties visées à l'annexe I de la Convention) s'engagent à réduire, au cours de la période d'engagement allant de 2008 à 2012, le total de leurs émissions des six principaux gaz à effet de serre ${ }^{(1)}$ en moyenne de 5,2 \% par rapport à cinq fois leur niveau de l'année 1990, pour les cinq secteurs suivants (sources) :

- énergie ;

- procédés industriels ;

- utilisation de solvants et d'autres produits ;

- agriculture ;

- déchets.

Le secteur de l'Utilisation des Terres, des Changements d'affectation des terres et de la Foresterie (UTCF-LULUCF en anglais) fait l'objet d'un traitement spécifique décrit plus bas.

Les pays en développement n'ont, quant à eux, pas d'objectifs chiffrés de réduction pour cette période (responsabilités communes mais différenciées).

Les engagements chiffrés de réduction des émissions figurent à l'annexe B du Protocole. La Communauté européenne doit ainsi réduire ses émissions de $8 \%$, le Japon de $6 \%$, la Fédération de Russie et la Nouvelle-Zélande doivent les stabiliser, etc.

Plusieurs Parties peuvent s'accorder pour remplir conjointement leurs engagements. Ainsi les quinze pays de l'Union européenne (avant le dernier élargissement) ont-ils conclu un accord interne de répartition des réductions, dit "accord de bulle". Selon cet accord, la France doit stabiliser ses émissions en 2008-2012 à leur niveau de 1990 et non pas les réduire de $8 \%$.

Le protocole institue trois mécanismes de flexibilité, qui peuvent être utilisés en complément des mesures prises au plan interne :

- l'échange de droits d'émission : «les Parties visées à l'annexe $B$ peuvent participer à des échanges de droits d'émission aux fins de remplir leurs engagements " ;

- la mise en œuvre conjointe (MOC) ;

- le mécanisme pour un développement propre (MDP).

Les deux derniers mécanismes permettent à un pays de l'annexe I de réaliser des projets de réduction d'émissions à l'extérieur de ses frontières (dans un pays développé ou en transition de l'annexe I pour la MOC, dans un pays en développement hors annexe I pour le MDP) et de les prendre en compte dans son bilan national.

La question des puits a été très controversée dès la négociation du protocole. L'enjeu était de savoir si les Parties visées à l'annexe I pouvaient comptabiliser leurs augmentations de stocks

(1) Il s'agit du dioxyde de carbone $\mathrm{CO}_{2}$, du méthane $\mathrm{CH}_{4}$, du protoxyde d'azote $\mathrm{N}_{2} \mathrm{O}$, des hydrofluorocarbures $\mathrm{HFC}$, des perfluocarbures PFC et de l'hexafluorure de soufre $\mathrm{SF}_{6}{ }_{6}$. Chacun de ces gaz a un potentiel de réchauffement global (PRG), ce qui permet une comptabilité globale en équivalents $\mathrm{CO}_{2}$. 
de carbone liées à l'UTCF pour remplir leurs engagements de réduction d'émissions de gaz à effet de serre et donc réduire d'autant leurs efforts internes. L'Union européenne, par exemple, a été longtemps hostile au recours aux puits.

Les opposants craignaient pour l'“intégrité environnementale” du Protocole (ne pas donner trop d'importance aux puits, au détriment des mesures spécifiques internes de réduction de consommation d'énergie), tandis que d'autres pays voyaient le recours aux puits en quelque sorte comme un quatrième mécanisme de flexibilité, nécessaire pour respecter leurs engagements.

Finalement, cette question est traitée par les articles 3.3 et 3.4 du Protocole (cf. encadré 2, cidessous). On notera que la dernière phrase de l'article 3.4, qui donne la possibilité de comptabiliser les puits lorsqu'il n'y a pas de changement d'affectation des terres dès la première période d'engagement 2008-2012, a été ajoutée lors de la dernière nuit de négociation et faisait partie du "paquet" de compromis final.

\section{Les Accords de Marrakech}

Les Conférences des Parties à la Convention successives ont adopté un certain nombre de décisions précisant les conditions d'application du Protocole. Formellement, ces décisions devront

\section{ENCADRÉ 2}

\section{PARAGRAPHES 3 ET 4 DE L’ARTICLE 3 DU PROTOCOLE DE KYOTO}

3.3. Les variations nettes des émissions de gaz à effet de serre par les sources et de l'absorption par les puits résultant d'activités humaines directement liées au changement d'affectation des terres et à la foresterie et limitées au boisement, au reboisement et au déboisement depuis 1990, variations qui correspondent à des variations vérifiables des stocks de carbone au cours de chaque période d'engagement, sont utilisées par les Parties visées à l'annexe I pour remplir leurs engagements prévus au présent article. Les émissions des gaz à effet de serre par les sources et l'absorption par les puits associées à ces activités sont notifiées de manière transparente et vérifiable et examinées conformément aux articles 7 et 8 .

3.4. Avant la première session de la Conférence des Parties agissant comme réunion des Parties au présent Protocole, chacune des Parties visées à l'annexe I fournit à l'Organe subsidiaire de conseil scientifique et technologique, pour examen, des données permettant de déterminer le niveau de ses stocks de carbone en 1990 et de procéder à une estimation des variations de ses stocks de carbone au cours des années suivantes. À sa première session, ou dès que possible par la suite, la Conférence des Parties agissant comme réunion des Parties au présent Protocole arrête les modalités, règles et lignes directrices à appliquer pour décider quelles activités anthropiques supplémentaires ayant un rapport avec les variations des émissions par les sources et de l'absorption par les puits des gaz à effet de serre dans les catégories constituées par les terres agricoles et le changement d'affectation des terres et la foresterie doivent être ajoutées aux quantités attribuées aux Parties visées à l'annexe I ou retranchées de ces quantités et pour savoir comment procéder à cet égard, compte tenu des incertitudes, de la nécessité de communiquer des données transparentes et vérifiables, du travail méthodologique du Groupe d'experts intergouvernemental sur l'évolution du climat, des conseils fournis par l'Organe subsidiaire de conseil scientifique et technologique conformément à l'article 5 et des décisions de la Conférence des Parties. Cette décision vaut pour la deuxième période d'engagement et pour les périodes suivantes. Une Partie peut l'appliquer à ces activités anthropiques supplémentaires lors de la première période d'engagement pour autant que ces activités aient eu lieu depuis 1990.

NB : les verbes "sont" (article 3.3 : obligatoire) et "peut" (article 3.4 : optionnel) ont été soulignés par les auteurs du présent article. 
être avalisées par la première Conférence des Parties agissant comme réunion des Parties au Protocole de Kyoto (COP-MOP1), qui se réunira du 28 novembre au 9 décembre 2005 à Montréal.

Le lecteur pourra se reporter au site internet de la Convention et en particulier au lien suivant, qui donne une vue globale des textes relatifs au secteur UTCF :

http://unfccc.int/methods_and_science/lulucf/items/1084.php

Les décisions des Conférences des Parties sont, elles, disponibles sur le lien :

http://unfccc.int/documentation/decisions/items/2646.php

La sixième Conférence des Parties à la Convention s'est réunie à La Haye en novembre 2000, mais n'a pas pu arriver à un accord sur les conditions de mise en œuvre du Protocole de Kyoto. Un accord politique a pu finalement être conclu à Bonn en juillet 2001, lors d'une session de reprise (dite "COP6-bis").

La septième Conférence des Parties s'est réunie à Marrakech en novembre 2001 et a adopté une quarantaine de décisions traduisant en termes techniques et juridiques l'accord politique de Bonn : ce sont les "accords de Marrakech".

La décision 11/CP.7 sur l'utilisation des terres, les changements d'affectation des terres et la foresterie précise les modalités d'application des articles 3.3 et 3.4 du Protocole et les conditions dans lesquelles les puits seront comptabilisés pendant la première période d'engagement.

\section{- Définitions}

Pour toute la durée de la première période d'engagement, chaque Partie visée à l'annexe I de la Convention devra retenir une définition des "forêts", en adoptant une seule et unique valeur minimale située entre 10 et $30 \%$ pour le couvert du houppier, entre 0,05 et 1 hectare pour la superficie et entre 2 et 5 mètres pour la hauteur des arbres à maturité.

- Boisement, reboisement, déboisement

(avec changement d'affectation des terres - article 3.3 du Protocole)

Aux termes de cet article, on devra (obligatoire) comptabiliser les variations (positives ou négatives) de stocks de carbone, entre 2008 et 2012, sur les terres non forestières boisées ou reboisées ${ }^{(2)}$ par action directe ${ }^{(3)}$ de l'homme depuis 1990 et sur les terrains déboisés (défrichés) depuis 1990.

La comptabilisation doit porter sur les cinq réservoirs de carbone suivants : biomasse aérienne, biomasse souterraine, litière, bois mort et carbone organique des sols.

Cet article a pour but d'encourager l'extension des surfaces boisées. Toutefois, comme la quantité de carbone déstockée sur un hectare de peuplement adulte défriché est très supérieure à la quantité de carbone stockée en cinq ans sur un hectare de jeune plantation, le mode de calcul peut aboutir à un bilan négatif pour un pays comme la France, alors même que les surfaces forestières augmentent (de l'ordre de 40000 ha/an en métropole, cf. IFN, 2005 et SCEES, 2005) et que les stocks de carbone augmentent (de l'ordre de $18 \mathrm{MtC} / \mathrm{an}$, cf. CARBOFOR, 2004) : il a donc été décidé que, pour la première période d'engagement, les éventuels débits pourraient être compensés sous certaines conditions.

(2) "On entend par "boisement" la conversion anthropique directe, en terres forestières, de terres qui n'avaient pas porté de forêts pendant au moins 50 ans (...). On entend par "reboisement" la conversion anthropique directe de terres non forestières en terres forestières (...) » : ce n'est donc pas le sens donné à ces deux expressions dans le langage courant des forestiers.

(3) Les Accords de Marrakech ne donnent pas de définition précise de cette expression. Après une interprétation stricte dans un premier temps, le guide des bonnes pratiques du GIEC laisse supposer que les colonisations "naturelles" (accrus) encouragées par les propriétaires forestiers pourraient être éligibles. 
- Gestion forestière

(sans changement d'affectation des terres - article 3.4 du Protocole)

Sous cet article, on pourra (optionnel) comptabiliser les augmentations de stocks de carbone entre 2008 et 2012 dans les forêts gérées, en se limitant aux activités directement dues à l'homme depuis 1990. Cette comptabilisation est plafonnée et la France pourra bénéficier à ce titre de l'annulation du débit éventuel de l'article 3.3 et d'un crédit de 880000 tC/an (soit 3,2 Mtéq $\left.\mathrm{CO}_{2} / \mathrm{an}\right)$ au maximum.

- Gestion des terres cultivées, gestion des pâturages, restauration du couvert végétal (sans changement d'affectation des terres - article 3.4 du Protocole)

Aux termes de cet article, on pourra (optionnel) comptabiliser les augmentations de stocks de carbone entre 2008 et 2012 liées à ces activités, en en soustrayant cinq fois l'effet en 1990 : c'est une comptabilité dite net-net dans le jargon des spécialistes.

Cette question des puits "agricoles" ne sera pas développée ici, l'article se limitant aux questions forestières.

Les Parties peuvent choisir de comptabiliser ou non, pour 2008-2012, chacune des quatre activités citées à l'article 3.4 du Protocole.

Il faut bien comprendre que ces dispositions sont le résultat d'un compromis - difficile adopté par consensus par près de deux cents pays souverains, cherchant à défendre chacun ses intérêts propres. Dans ces conditions, il serait illusoire de leur rechercher une logique interne ou une rationalité technique fortes. Le résultat est au contraire d'une complexité certaine !

\section{Le guide des bonnes pratiques du GIEC}

Le Groupe intergouvernemental d'Experts sur le Changement climatique (GIEC) a publié fin 2003 un volumineux Guide des bonnes pratiques sur l'utilisation des terres, les changements d'affectation des terres et la foresterie (IPCC, 2004), qui est en quelque sorte la circulaire d'application du Protocole et des Accords de Marrakech pour le secteur UTCF.

Quatre experts français (Dominique Arrouays, Jean-Claude Germon, Denis Loustau, Gérôme Pignard) ont contribué à la rédaction du guide.

La neuvième Conférence des Parties a décidé que les Parties devaient utiliser ce guide pour l'établissement de leurs inventaires annuels d'émissions/absorptions de gaz à effet de serre en application de la Convention (décision 13/CP.9).

La dixième Conférence des Parties a proposé que les Parties au Protocole utilisent le guide pour fournir les informations requises pour l'application des articles 3.3 et 3.4 du Protocole (projet de décision -/CMP.1 annexé à la décision 15/CP.10).

\section{Les puits dans le MDP}

La neuvième Conférence des Parties a adopté les modalités et procédures de prise en compte des activités de boisement et de reboisement au titre du Mécanisme pour un Développement propre (MDP) au cours de la première période d'engagement du Protocole de Kyoto (décision 19/CP.9).

La dixième Conférence des Parties a adopté les modalités et procédures simplifiées pour les “petits" projets (décision 14/CP.10). 
Cette question, qui justifierait la rédaction d'un article séparé, est signalée pour mémoire et ne sera pas développée ici.

\section{Les textes communautaires}

La décision $n^{\circ}$ 280/2004/CE du Parlement européen et du Conseil du 11 février 2004 relative à un mécanisme pour surveiller les émissions de gaz à effet de serre dans la Communauté et mettre en œuvre le Protocole de Kyoto, d'une part, et, d'autre part, la décision n 166/2005/CE de la Commission du 10 février 2005 qui en fixe les modalités d'exécution précisent les modalités d'établissement et le calendrier de transmission par les États membres à la Commission européenne des informations requises par le Protocole de Kyoto.

Ces deux décisions sont immédiatement applicables en France. Ainsi, la France devait-elle :

- pour le 31 décembre 2005, avoir défini son système national d’inventaire des émissions/absorptions de gaz à effet de serre : " prendre et maintenir les dispositions institutionnelles, juridiques et de procédure nécessaires, réparties selon qu'il conviendra entre les organismes publics et d'autres entités » permettant d'estimer les émissions anthropiques par les sources et l'absorption par les puits de tous les gaz à effet de serre non réglementés par le Protocole de Montréal sur la protection de la couche d'ozone ;

- pour le 15 janvier 2006, avoir transmis à la Commission européenne son rapport initial sur les "quantités attribuées ». Pour le secteur UTCF, la France devait fournir la série complète de ses inventaires d'émissions/absorptions depuis 1990, décider quelles activités elle choisit de comptabiliser au titre de l'article 3.4 du Protocole, définir les "forêts » et les "forêts gérées ", préciser comment elle détermine les superficies consacrées aux diverses activités, opter pour une comptabilisation annuelle ou sur l'ensemble de la période d'engagement... Le rapport devait également comprendre un aperçu du système national d'inventaire et du système de registres.

\section{LA MISE EN GUVRE POUR LES FORÊTS FRANÇAISES}

\section{L’inventaire des émissions/absorptions}

En France, l'inventaire des émissions anthropiques par les sources et des absorptions par les puits de gaz à effet de serre, requis par la convention-cadre, est de la responsabilité du ministère chargé de l'Écologie, qui en a confié la réalisation (maîtrise d'œuvre) au Centre interprofessionnel technique d’Études de la Pollution atmosphérique (CITEPA).

En 2003, les émissions totales françaises se sont élevées à 557 millions de tonnes équivalentes à du $\mathrm{CO}_{2}\left(\mathrm{MtéqCO}_{2}\right)$, réparties comme suit (CITEPA, 2004 et 2005) :

- transports : $27 \%$

- résidentiel tertiaire : $18 \%$

- industrie manufacturière : $20 \%$

— industrie de l'énergie : $13 \%$

- agriculture : $19 \%$

- traitement des déchets : $3 \%$

L'agriculture produit peu de dioxyde de carbone $\left(\mathrm{CO}_{2}\right)$, mais beaucoup de méthane $\left(\mathrm{CH}_{4}\right)$ et de protoxyde d'azote $\left(\mathrm{N}_{2} \mathrm{O}\right)$ dont le pouvoir de réchauffement global est plus élevé (voir encadré 3, p. 486). 


\section{ENCADRÉ 3}

\section{LES ÉMISSIONS DE GAZ À EFFET DE SERRE DU SECTEUR AGRICOLE ET FORESTIER EN 2003 EN FRANCE}

\begin{tabular}{|c|c|c|}
\hline & & t éq $\mathrm{CO}_{2}$ \\
\hline 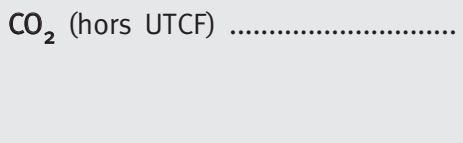 & 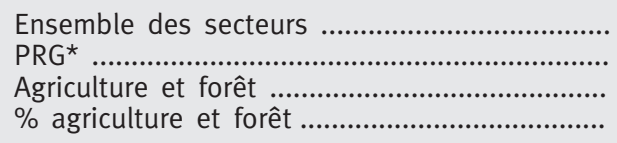 & $\begin{array}{l}408 \\
1 \\
10 \\
3 \%\end{array}$ \\
\hline $\mathrm{CH}_{4}$ & 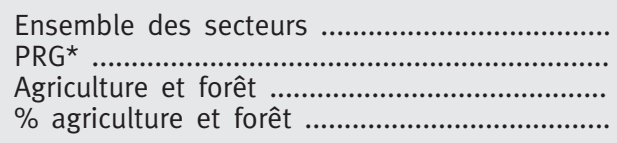 & $\begin{array}{l}61 \\
21 \\
43 \\
70 \%\end{array}$ \\
\hline $\mathrm{N}_{2} \mathrm{O}$ & 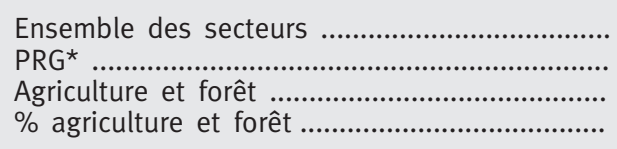 & $\begin{array}{l}75 \\
310 \\
57 \\
76 \%\end{array}$ \\
\hline 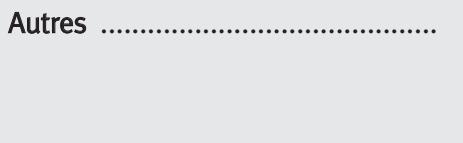 & 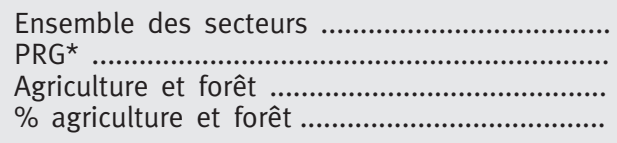 & $\begin{array}{l}14 \\
- \\
-\end{array}$ \\
\hline 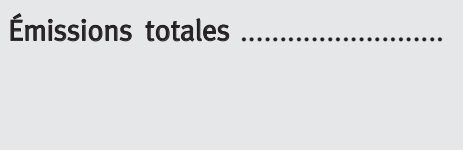 & 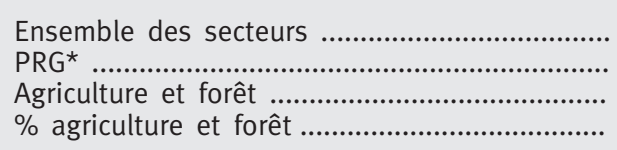 & $\begin{array}{l}558 \\
110 \\
20 \%\end{array}$ \\
\hline Puits nets (UTCF) .............................. & 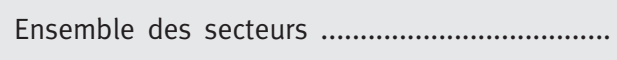 & -53 \\
\hline 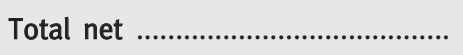 & 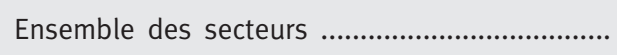 & 505 \\
\hline
\end{tabular}

* Pouvoir de réchauffement global. À titre d'exemple, l'effet sur le réchauffement, cumulé sur une période de 100 ans, produit par l'émission d'une tonne de méthane dans l'atmosphère est 21 fois celui d'une tonne de dioxyde de carbone.

Depuis 1990, les émissions des sols agricoles $\left(\mathrm{N}_{2} \mathrm{O}\right)$ diminuent (réduction des intrants). Les émissions de la fermentation entérique $\left(\mathrm{CH}_{4}\right)$ et des déjections animales $\left(\mathrm{CH}_{4}\right.$ et $\left.\mathrm{N}_{2} \mathrm{O}\right)$ diminuent légèrement (régression du cheptel). La consommation d'énergie $\left(\mathrm{CO}_{2}\right)$ est stable.

Le secteur de l'utilisation des terres, des changements d'affectation des terres et de la foresterie (UTCF) a représenté, quant à lui, un puits de $53 \mathrm{MtéqCO}_{2}$, équivalant à près de $10 \%$ des émissions brutes.

\section{Le groupe de travail UTCF}

Dès 2002, après la conclusion des Accords de Marrakech, le ministère de l'Agriculture (DERF) a mis en place un groupe de travail informel, regroupant les quatre co-auteurs français du guide 
des bonnes pratiques du GIEC et tous les ministères et organismes techniques concernés, sur les conditions pratiques de mise en œuvre de ces décisions, en métropole et dans les départements d'Outre-Mer. Il a lancé une étude sur l'utilisation d'images satellitaires en Guyane, en partenariat avec l'Agence spatiale européenne.

Des propositions ont ainsi été rédigées et adressées début 2004 à la Mission interministérielle de l'Effet de Serre (MIES), pour nourrir les discussions interministérielles (MAAPAR-IFN, 2004). Elles portent sur les points suivants :

- Définition de la forêt (articles 3.3 et 3.4 du Protocole de Kyoto)

Comme noté ci-dessus, les Parties visées à l'annexe I de la Convention doivent adopter une seule et unique valeur minimale pour le couvert du houppier, la superficie et la hauteur des arbres.

Ce choix pose un problème particulier à la France, qui est le seul pays de l'annexe I dont un tiers de la forêt est une forêt tropicale. Il n'existe pas à l'heure actuelle de dispositif permettant de suivre les défrichements et reboisements sur les 7,5 millions d'ha de forêt guyanaise avec une précision de l'ordre de l'hectare. Le recours à des images satellitaires sera nécessaire.

La proposition est de retenir les mêmes seuils que pour l'évaluation des ressources forestières faite par la FAO, c'est-à-dire :

- taux de couvert : $10 \%$

- surface unitaire d'un seul tenant : 0,5 ha

- hauteur : $5 \mathrm{~m}$

- Définition des forêts gérées (article 3.4 du Protocole de Kyoto)

Retenir l'activité "gestion forestière" au titre de l'article 3.4 du Protocole de Kyoto apparaît bénéfique pour la France et permettrait d'annuler un éventuel débit de l'article 3.3, tout en obtenant un crédit de 3,2 MtéqCO $/$ an. Les difficultés de comptabilisation et de mise en œuvre n'apparaissent pas insurmontables.

Il est donc proposé de retenir comme "forêts gérées" les forêts qui font l'objet d'opérations de gestion forestière visant à administrer les fonctions écologiques, économiques et sociales de la forêt. Le terme "opération de gestion forestière" recouvre entre autres les actions de coupes ou de travaux forestiers, les actions de planification forestière, d'accueil du public en forêt ou de protection des écosystèmes forestiers. Seules les forêts exclusivement soumises aux processus naturels, en raison notamment d'une accessibilité limitée, sont considérées comme non gérées : c'est entre autres le cas des forêts de haute montagne ou de la mangrove guyanaise.

Cette définition retiendrait donc comme "forêts gérées" les surfaces suivantes :

- en métropole, 14,6 millions d'ha, soit $94 \%$ des forêts, ce qui correspond d'ailleurs à la surface des formations boisées de production de l'IFN ;

- en Guadeloupe, en Martinique et à la Réunion, la totalité des superficies boisées ressortant de l'enquête Teruti (186 ooo ha);

- en Guyane, les forêts de la bande littorale (500 000 ha), les forêts aménagées ou à aménager par l'ONF (800 000 ha) et les 400000 ha de forêts grevées de droits d'usage à la culture sur abattis itinérants, soit un total de 1,7 million d'ha sur 7,5 millions d'ha (23\%) (4).

(4) Cette proposition initiale sera vraisemblablement revue à la baisse. 
- Système d'inventaire et localisation géographique des activités (articles 3.3 et 3.4 du Protocole de Kyoto)

Après des débats difficiles portant sur le géo-référencement des activités, les Accords de Marrakech autorisent finalement l'identification et l'évaluation par échantillonnage des activités au sein de limites juridiques, administratives ou écologiques, englobant différents types d'activités ainsi que d'autres usages du sol. Bien qu'un inventaire exhaustif du territoire soit théoriquement plus satisfaisant pour suivre l'utilisation et les changements d'affectation des sols, il apparaît que la France ne dispose pas, depuis 1990, d'un outil adapté. La précision de l'inventaire CORINE Land Cover, par exemple, est largement insuffisante.

En conséquence, le groupe de travail propose que la France comptabilise ses émissions/absorptions dans les vingt-deux régions de métropole et les quatre départements/régions d'Outre-Mer. À l'intérieur de chacune de ces vingt-six entités, l'utilisation et les changements d'affectation des sols seront suivis de manière statistique. Les grandeurs recherchées seront observées sur un échantillon représentatif du territoire et extrapolées à l'ensemble de l'entité par application des méthodes habituelles des statisticiens qui permettent notamment de connaître la variance et la précision des estimations de surfaces.

\section{Les sources statistiques}

Deux sources statistiques sont disponibles et seront utilisées pour suivre l'utilisation et les changements d'affectation des sols ; leurs protocoles ont été modifiés et harmonisés en 2005 pour répondre aux recommandations du guide des bonnes pratiques du GIEC et ainsi aux engagements de la France.

La première est l'enquête sur l'utilisation du territoire (SCEES, 2005) qui observe l'occupation et l'usage de l'ensemble du territoire : agricole, forestier, naturel et urbanisé. En métropole, on dispose de trois séries historiques : Teruti 1982-1990, Teruti 1992-2004 et Teruti-Lucas à partir de 2005. L'échantillon représentatif du territoire comprend 150000 points regroupés en 15000 segments. Dans les départements d'Outre-Mer, on dispose de la série historique TERUTIDOM 1992-2004 pour la Guadeloupe, la Martinique et la Réunion. À partir de 2005 pour la Martinique et la Réunion, de 2006 pour la Guadeloupe et la bande côtière de la Guyane, commence la série historique Teruti-Lucas réalisée selon la même méthodologie qu'en métropole.

La deuxième est l'Inventaire forestier national qui observe l'occupation forestière de manière beaucoup plus approfondie, car c'est sa vocation, mais les autres occupations plus superficiellement. Jusqu'en 2004, l'inventaire était réalisé à une périodicité de dix ans dans chaque département. À partir de 2005, l'inventaire est actualisé chaque année sur l'ensemble du territoire métropolitain, un dixième des points de chaque département étant observé annuellement. L'Inventaire forestier national ne concerne pas les départements/régions d’Outre-Mer.

\section{Impact de l'intensification de la récolte de bois sur le crédit carbone de l'activité gestion forestière}

(article 3.4 du Protocole de Kyoto)

Un des objectifs de la politique forestière française est d'intensifier la sylviculture et d'accroître les prélèvements de bois. Mais la France peut obtenir des crédits, plafonnés à 880000 tC/an, si ses stocks de carbone augmentent dans les forêts gérées. Ces deux points peuvent apparaître contradictoires!

En réalité, le plafond ci-dessus est censé exclure du stockage total de carbone dans les forêts gérées la partie de ce stockage qui résulterait d'actions de gestion antérieures à 1990 ou qui 
serait la conséquence de modifications environnementales (augmentation du taux de $\mathrm{CO}_{2}$ dans l'atmosphère, dépôts azotés). Lors des négociations à Bonn en 2001, un abattement forfaitaire de $85 \%$ a ainsi été appliqué pour calculer le plafond.

Le taux de prélèvement dans les forêts de métropole, pour 1998-2002, n'est que de 69 \% de la production courante nette (IFN, 2005). Le puits est estimé à $18 \mathrm{MtC} / \mathrm{an}{ }^{(5)}$ (CARBOFOR, 2004). Si elle le souhaite, la France peut donc considérablement accroître ses prélèvements, sans risquer de perdre son crédit de 880000 tonnes par an pour la première période d'engagement.

Ce point est particulièrement important pour le développement d'une filière bois-énergie en France : la récolte de bois supplémentaire sera comptabilisée au titre de l'article 3.3 ou 3.4 du Protocole au moment de la coupe, le puits forestier diminuera mais cela ne changera pas, du fait du plafonnement, le crédit obtenu. Les émissions de gaz à effet de serre ne seront pas comptabilisées au moment de la combustion du bois, puisqu'elles l'auront déjà été au moment de la coupe. À l'inverse, la combustion évitée de combustible fossile se traduira bien par une baisse des émissions du secteur considéré (énergie, industrie) : le bilan vis-à-vis du Protocole de Kyoto est donc positif ${ }^{(6)}$.

\section{Les mesures adoptées ou prévues}

Le Gouvernement a adopté en juillet 2004 le "Plan Climat" (MIES, 2004), dont le chapitre 5 "Agriculture durable et forêts" prévoit un certain nombre de mesures liées au Protocole de Kyoto : puits de carbone, valorisation des produits issus de la biomasse (bois énergie, biocarburants, bois dans la construction).

À la date de rédaction de cet article (septembre 2005), le choix des activités à retenir au titre de l'article 3.4 du Protocole et les choix relatifs aux définitions doivent encore faire l'objet d'arbitrages interministériels, avant que la France adresse à la Commission européenne son rapport sur les quantités attribuées.

Un groupe de travail a été mis en place le 30 mai 2005 par le ministère de l’Écologie et du Développement durable, pour définir le détail des options méthodologiques, en conformité avec le guide des bonnes pratiques.

Un des points difficiles, et certainement onéreux, sera la mise en place d'un dispositif adapté à la forêt guyanaise.

Un point central de la méthodologie devra permettre de distinguer (cf. note de bas de page (2), p. 483):

- les coupes rases dans une forêt gérée (article 3.4) des défrichements (article 3.3) ;

- les plantations dans une forêt gérée (article 3.4) des boisements et reboisements (article 3.3).

Sur le terrain, face à une parcelle coupée à blanc, l'enquêteur ne peut pas savoir d'avance si elle sera replantée ou non : il faudra donc suivre la situation de la parcelle, non seulement d'une année sur l'autre, mais aussi sur une période plus longue, de l'ordre de cinq ans (7).

\footnotetext{
(5) Hors variation du stock de carbone dans la matière organique des sols forestiers, non évaluée à ce jour.

(6) Alors qu'il est neutre vis-à-vis de la Convention-cadre, le $\mathrm{CO}_{2}$ provenant du bois ou des combustibles fossiles étant comptabilisé de la même manière.

(7) Même si cela peut apparaître a priori surprenant, il n'existe pas en France d'inventaire permanent des défrichements et des reboisements. L'évaluation des surfaces défrichées ou reboisées en métropole et dans les DOM depuis 1990 à partir des sources statistiques citées ci-dessus pose des problèmes méthodologiques complexes mais qui sont cependant en voie de résolution.
} 


\section{Quels bénéfices ou quelles contraintes pour les propriétaires forestiers et les industriels du bois ?}

Il faut ici distinguer les bénéfices ou contraintes ressortant de la première période d'engagement de ceux qui pourraient apparaître au cours des périodes suivantes.

Au cours de la première période, les bénéfices semblent assez limités. En effet, en application des Accords de Marrakech, un pays de l'annexe I ne peut pas vendre sur le marché international des droits d'émission les crédits carbone ("unités d'absorption") obtenus au titre des articles 3.3 et 3.4. Les choix que la France fera relativement à la vente individuelle des crédits, et en particulier l'éventualité d'un traitement différencié pour le secteur forestier du fait de ses spécificités, seront donc déterminants ${ }^{(8)}$.

Cependant, des projets de boisement de terres non forestières pourront éventuellement être mis en œuvre en France par des États de l'annexe I, ou par des entreprises de ces États, au titre de la mise en œuvre conjointe (MOC). Ces boisements apporteront des crédits d'émission à ces États, mais pas à la France. Néanmoins, ils participeront à l'augmentation de la surface boisée de la France et seront portés à son crédit au titre de l'article 3.3.

En dehors de ce cas particulier, il serait également logique que les aides existantes au boisement des terres agricoles soient pérennisées, voire rendues plus attractives, tout en devenant plus différenciées en faveur des régions peu boisées. En effet, les régions qui se boisent sont souvent déjà les plus boisées. L'effet de serre doit donc être intégré dans une approche globale prenant également en compte les objectifs d'aménagement du territoire.

Nous avons vu plus haut que les règles comptables du Protocole de Kyoto favorisent le développement d'une filière bois-énergie. On peut donc attendre d'une augmentation de la demande de bois-énergie une amélioration du revenu des propriétaires et de l'activité des entreprises d'exploitation forestière. En revanche, les variations de stocks de bois dans les constructions ne sont pas prises en considération durant la première période d'engagement. Le Protocole n'aura donc pas d'effet direct sur la consommation de bois d'œuvre et de panneaux.

Au chapitre des contraintes, la seule mesure éventuellement envisageable serait le renforcement de la législation sur le défrichement. En effet, tout défrichement, même compensé par un boisement sur un autre terrain, est, au titre de l'article 3.3, porté au débit du pays où il a été réalisé. Rappelons toutefois que le Code forestier français est déjà très contraignant sur ce point et que la prise en compte de l'activité gestion forestière dans le cadre de l'article 3.4 permettrait d'annuler l'éventuel débit ${ }^{(9)}$ de la France au titre de l'article 3.3.

Au-delà de la première période d'engagement, les hypothèses sont nombreuses et les auteurs ne prétendent pas déchiffrer l'avenir. Comme nous l'avons vu, la substitution du bois, éco-matériau ou bio-énergie, à des matériaux dont la fabrication est plus coûteuse en énergie ou à des combustibles fossiles, participe à la lutte contre les changements climatiques. Il en est de même de la séquestration du carbone en forêt et hors forêt sous forme de bois matériau. Les mécanismes de Kyoto mettent en place des moyens financiers nouveaux dont devrait pouvoir profiter l'ensemble de la filière française, dans la mesure où elle saura s'organiser pour faire valoir ses arguments lors de l'adoption puis de l'évolution du dispositif national.

(8) Étude de la Caisse des Dépôts et Consignations (CDC) sur les "projets domestiques" en cours.

(9) À ce stade, les auteurs s'abstiendront volontairement de tout pronostic sur le bilan final de l'article 3.3, qui serait tout à fait prématuré, tant les incertitudes sont grandes : prise en compte ou non des accrus en métropole, niveau réel des défrichements en Guyane, etc. 


\section{LES NÉGOCIATIONS FUTURES}

\section{Le stockage de carbone dans les produits en bois}

Le compromis adopté à Marrakech pour la première période d'engagement 2008-2012 prend en compte la séquestration de carbone dans les forêts et les sols. Il favorise la valorisation énergétique du bois, dans la mesure où les émissions liées à la combustion de biomasse ne sont pas comptabilisées au titre du Protocole.

En revanche, il ne valorise pas les efforts visant à une utilisation accrue du bois matériau. Même si tous les pays sont d'accord sur le fait qu'il serait légitime et souhaitable de comptabiliser le carbone séquestré dans les produits en bois, les avis divergent quant à l'approche à retenir. Schématiquement, trois approches sont discutées depuis dix ans, qui sont sommairement présentées dans l'encadré 4 (ci-dessous) et donnent, pour un pays donné, des résultats différents, selon la façon dont sont comptabilisés les produits faisant l'objet d'un commerce extérieur (Chaudron et Loisel, 2003).

\section{ENCADRÉ 4 \\ LES TROIS APPROCHES \\ DE COMPTABILISATION DES PRODUITS EN BOIS}

- La première approche, dite des variations de stocks, consiste à comptabiliser pour chaque pays la variation de stocks de carbone d'une part dans les forêts et d'autre part dans les produits en bois d'une période sur l'autre. Les variations de stocks, reflétant des émissions ou des absorptions nettes de dioxyde de carbone, sont donc attribuées au pays où elles ont lieu et quand elles ont lieu.

Cette approche est plutôt favorable aux pays importateurs car, à la différence de la seconde méthode, l'échange d'un produit entre un pays et un autre est indirectement compté comme une émission du pays exportateur et une absorption du pays importateur.

- La deuxième approche, dite des flux atmosphériques, consiste à comptabiliser d'une part les absorptions nettes en forêt (l'exploitation du bois n'étant pas considérée comme une émission) et d'autre part les émissions en fin de vie des produits.

Cette approche attribue donc bien les émissions et absorptions où et quand elles ont lieu. Elle est plutôt favorable aux pays exportateurs.

- La troisième approche, dite de la production, attribue l'absorption en forêt au pays où se situe la forêt et l'émission en fin de vie des produits en bois quand elle a lieu, mais pour le pays où le bois a été produit. Un pays devrait ainsi comptabiliser des émissions qui ont lieu hors de son territoire et sur lesquelles il n'a aucune prise, ce qui est un peu difficile à justifier et à mettre en pratique.

Les discussions sont ouvertes pour la deuxième période d'engagement et l'Union européenne a déjà marqué à plusieurs reprises sa préférence de principe pour la méthode des variations de stocks. En tout état de cause, il n'existe pas actuellement en France de dispositif de suivi de l'évolution des produits bois, depuis leur production jusqu'à leur combustion ou leur décomposition en décharge. Leur durée de vie varie de quelques semaines pour le papier journal à quelques siècles pour les plus belles charpentes... 


\section{L’après 2012}

Les discussions générales sur le futur régime international pour le climat, ou "post-2012" dans le jargon des négociations internationales, ont d'ores et déjà commencé. Un séminaire informel d'experts gouvernementaux s'est réuni en mai 2005 à Bonn à ce sujet. L'objectif "politique" général est de pouvoir y associer :

- d'une part les États-Unis, plus gros émetteur de gaz à effet de serre de la planète, mais qui n'ont pas ratifié le Protocole de Kyoto ;

- d'autre part les grands pays émergents, comme la Chine, l'Inde ou le Brésil, qui n'avaient pas d'engagement de réduction de leurs émissions entre 2008 et 2012.

Quelle sera la place du secteur UTCF dans le futur régime ? Pour la première période d'engagement, l'accent a surtout été mis, comme on l'a vu ci-dessus, sur la séquestration du carbone dans les forêts et dans les sols.

Deux autres pistes seront sans aucun doute étudiées :

- la substitution de produits agricoles ou forestiers à des carburants fossiles ou à des matières premières : biocarburants, biomatériaux, utilisation énergétique des biomasses, bois construction, etc.

- la réduction des émissions : la déforestation tropicale représente $20 \%$ des émissions mondiales de gaz à effet de serre $(\mathrm{ONF}, 2002)$ et a lieu dans des pays qui n'ont pas d'engagement de réduction pour 2008-2012. La question dite de la "déforestation évitée" dans les pays en développement, qui avait déjà été discutée, mais pas retenue, en 2001 (Riedacker, 2004), sera de nouveau à l'ordre du jour de la $11^{\mathrm{e}}$ Conférence des Parties, en novembre-décembre 2005 à Montréal, à la demande de la Papouasie-Nouvelle-Guinée et du Costa Rica.

\section{CONCLUSIONS}

Le Protocole de Kyoto, en discussion depuis 1995, a été adopté en 1997 ; les Accords de Marrakech ont été conclus en 2001 ; le Protocole est entré en vigueur en février 2005 ; en fin d'année 2005, chaque État signataire devait avoir défini ses choix pour la première période d'engagement, de 2008 à 2012.

On est donc dans un processus long, de plus de dix années, qui a connu des hauts et des bas (lorsque les États-Unis ont annoncé qu’ils ne ratifieraient pas le Protocole ou lorsque la Fédération de Russie a envoyé des signaux contradictoires sur ses intentions de le ratifier ou non) et qui donne l’impression de s'accélérer brusquement, lorsque des décisions sont à prendre pour la première période d'engagement...

Pour la deuxième période d'engagement, de 2013 à 2017, les négociations commencent. C'est demain, en 2006, en 2007, que tout se jouera et qu'il faudra s'organiser, dans l'intérêt de notre filière forêt-bois.

Les questions posées sont nombreuses. Les incertitudes sont élevées. Comment les peuplements forestiers vont-ils évoluer face aux changements annoncés : canicules, tempêtes ? Comment répondre à la demande de bois-énergie ? Quel sera le bilan en Guyane ? Et, plus généralement, quelle gestion voulons-nous pour nos forêts tropicales ? Quelle stratégie d'adaptation de nos forêts au changement climatique allons-nous définir, en termes de sylviculture ou de choix des essences et des provenances? 
Une dernière remarque, mais pas la moindre, est que si le réchauffement s'intensifie comme les modèles le prédisent, n’y a-t-il pas le risque d'un déstockage massif des sols et peuplements forestiers ?
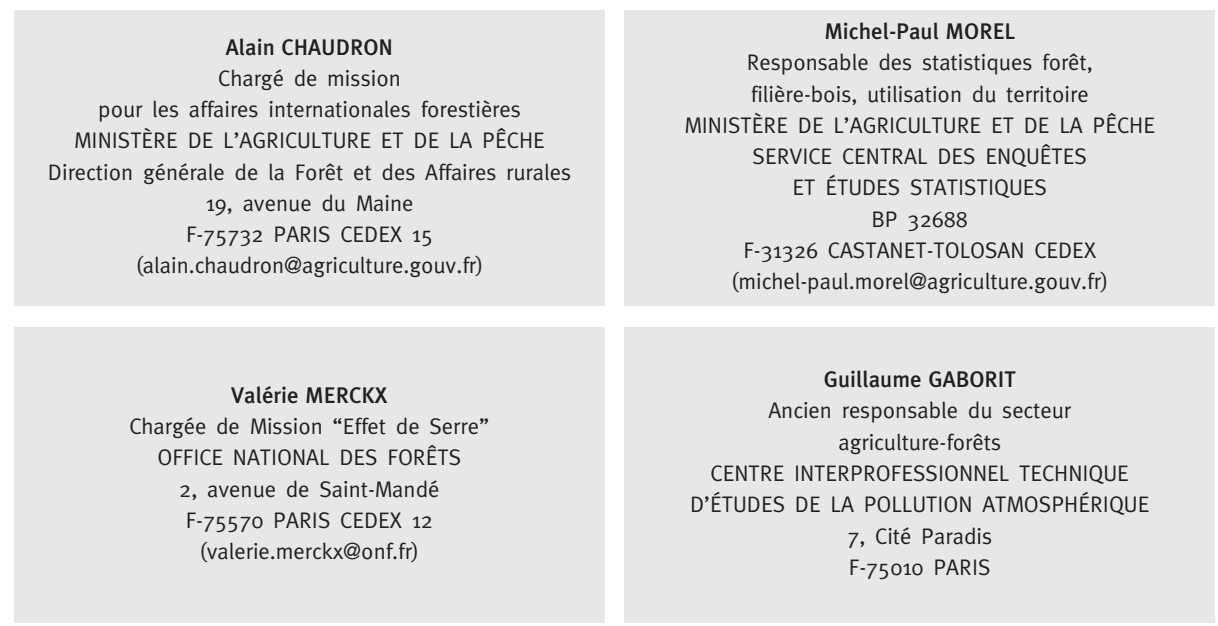

\section{Remerciements}

Les auteurs remercient vivement Gérôme Pignard et Arthur Riedacker, pour leur relecture critique du manuscrit.

\section{BILAN DE LA CONFÉRENCE DE MONTRÉAL 28 novembre - 9 décembre 2005}

\section{Les acquis de la Conférence sur le régime actuel}

La Conférence de Montréal a permis d’adopter dans leur intégralité les Accords de Marrakech relatifs au fonctionnement du Protocole de Kyoto.

Les négociations sur les mécanismes de flexibilité ont également abouti. La consolidation des méthodes de gestion du mécanisme pour un développement propre (MDP) a été approuvée, autorisant à terme un renforcement significatif de l'équipe (jusqu'à 30 personnes permanentes), ce qui permettra de traiter dans de meilleures conditions le plan de charge 2006 qui compte actuellement environ 300 projets de MDP et 80 projets de méthodologie de calcul. Néanmoins, ce renforcement n'a été possible que par des annonces de financement, coordonnées par la présidence canadienne, pour un montant d'environ 8 millions de USD, en attendant le relais d'un autofinancement dégagé par les prélèvements sur les projets enregistrés.

Enfin, les discussions relatives à l'utilisation des règles et procédures MDP destinées à assurer un démarrage rapide des projets du mécanisme de mise en œuvre conjointe (MOC - principalement dans les pays à économie en transition) ont également été finalisées. 


\section{L’issue des négociations sur le futur régime}

À l'issue de consultations longues et intenses de la présidence canadienne, un accord a pu être obtenu sur trois documents concernant la négociation du futur régime après 2012 :

- le premier document traite des engagements pour la période après 2012 des pays industrialisés de l'annexe I, dans le cadre de l'article 3.9 du Protocole de Kyoto. II prévoit que soit instauré un groupe de travail ad hoc qui se réunira dès mai 2006, et qui devra terminer ses travaux dans des délais qui doivent permettre d'éviter toute interruption du régime du Protocole de Kyoto en 2012. Cette décision sécurise le marché de carbone lancé par le Protocole de Kyoto en envoyant un signal clair sur la poursuite du Protocole après 2012 ;

- le deuxième, adopté dans le cadre de la Conférence des Parties à la Convention (189 parties, dont les États-Unis), a pour objectif d'instaurer un dialogue visant à définir de nouvelles approches qui doivent aider et encourager les États à prendre les mesures appropriées pour lutter contre le changement climatique et contre ses impacts. Elle jette les bases d'un dialogue informel et non contraignant sur les deux prochaines années. Isolés, les États-Unis se sont joints dans les dernières heures à la négociation : les quelques amendements proposés n'ont pas remis en question l'accord conclu auparavant entre le groupe des 77 et les autres pays industrialisés ;

- le troisième document concerne l'article 9 du Protocole de Kyoto visant à un réexamen global du Protocole, à la lumière des dernières études scientifiques et socio-économiques. II appelle notamment les Parties à présenter des soumissions avant les prochaines COP et COP/MOP.

Extrait d'un document de travail du Secrétariat général des Affaires européennes (SGAE) du 10 janvier 2006.

\section{BIBLIOGRAPHIE}

AGENCE SPATIALE EUROPÉENNE / EUROPEAN SPACE AGENCY. - Services supporting the UNFCCC and Kyoto Protocol, Reporting on Land Use, Land-Use Change and Forestry. - Agence spatiale européenne, 2004. $-8 \mathrm{p} .+$ fiche.

CAISSE DES DÉPÔTS ET CONSIGNATIONS (CDC), OFFICE NATIONAL DES FORÊTS (ONF). - "Puits de carbone domestiques" : quel intérêt pour la France ? - CDC ; ONF, juillet 2005. - 5 p.

CARBOFOR. - Séquestration de carbone dans les grands écosystèmes forestiers en France, rapport final. 2004. - $134 \mathrm{p}$.

CHAUDRON (A.). - Le Protocole de Kyoto et les puits de carbone. - Forêts de France, $\mathrm{n}^{\circ}$ 450, janvier-février 2002, pp. 37-39.

CHAUDRON (A.), LOISEL (C.). - La forêt, le bois et l'effet de serre. - La Jaune et la Rouge, $\mathrm{n}^{\circ}$ 581, janvier 2003, pp. 6-9.

CENTRE INTERPROFESSIONNEL TECHNIQUE D’ÉTUDES DE LA POLLUTION ATMOSPHÉRIQUE (CITEPA). - Évaluation des puits de $\mathrm{CO}_{2}$ suivant la nouvelle méthode préconisée par le FIEC. - Paris : CITEPA, 1999. 22 p. + annexes.

CENTRE INTERPROFESSIONNEL TECHNIQUE D’ÉTUDES DE LA POLLUTION ATMOSPHÉRIQUE (CITEPA). - Inventaire des émissions de gaz à effet de serre en France au titre de la Convention cadre des Nations Unies sur les changements climatiques (CCNUCC/crf). - Paris : CITEPA, décembre 2004. - 127 p. + annexes.

CENTRE INTERPROFESSIONNEL TECHNIQUE D’ÉTUDES DE LA POLLUTION ATMOSPHÉRIQUE (CITEPA). - Inventaire des émissions de polluants atmosphériques en France. - Paris : CITEPA - Séries sectorielles et analyses étendues (SECTEN), février 2005. - $247 \mathrm{p}$.

COMITÉ NATIONAL POUR LE DÉVELOPPEMENT DU BOIS (CNDB), NORDIC TIMBER COUNCIL (NTC). - Le Rôle du bois dans la lutte contre les changements climatiques. - CNDB ; NTC, 2005. - $12 \mathrm{p}$.

DUPOUEY (J.-L.), PIGNARD (G.) et al. . - Stocks et flux de carbone dans les forêts françaises. In : Bilan et gestion des gaz à effet de serre dans l'espace rural. - Comptes rendus de l'Académie d'Agriculture de France, vol. 85, n 6, 1999. 
DUPOUEY (J.-L.), PIGNARD (G.). - Quelques problèmes posés par l'évaluation des stocks et flux de carbone forestiers au niveau national. - Revue forestière française, vol. LIII, n 3-4, 2001, pp. 294-300.

FAO. - Climate change and the forest sector. - FAO Forestry Paper, $n^{\circ} 144,2004,60 \mathrm{p}$.

HÖHNE (N.), WARTMANN (S.), HEROLD (A.), FREIBAUER (A.). - The rules for land use, land use change and forestry under the Kyoto protocol. - Lessons learned for the future climate negotiations, 2004. - $29 \mathrm{p}$.

INTERGOVERNMENTAL PANEL ON CLIMATE CHANGE (IPCC). - Land use, land-use change and forestry. IPCC, 2000. - $377 \mathrm{p}$.

INTERGOVERNMENTAL PANEL ON CLIMATE CHANGE (IPCC). - Good Practise Guidance for Land use, land-use change and forestry. - IPCC, 2004. - 600 p.

INVENTAIRE FORESTIER NATIONAL (IFN). - La Forêt française : un puits de carbone ? Son rôle dans la limitation des changements climatiques. - L'IF, $\mathrm{n}^{\circ} 7$, mars 2005, $8 \mathrm{p}$.

INVENTAIRE FORESTIER NATIONAL (IFN). - Les indicateurs de gestion durable des forêts françaises 2005, version provisoire. - Inventaire forestier national, juin 2005.

JUNG (M.). - The history of sinks - An analysis of negotiating positions in the climate regime. - HWWA discussion paper $\mathrm{n}^{\circ}$ 293, 2004.

MINISTÈRE DE L'AGRICULTURE, DE L'ALIMENTATION, DE LA PÊCHE ET DES AFFAIRES RURALES (MAAPAR), INVENTAIRE FORESTIER NATIONAL (IFN). - Définitions et options pour la mise en œuvre du Protocole de Kyoto en France (secteur UTCF). - Cinq notes techniques, 2004.

MISSION INTERMINISTÉRIELLE DE L'EFFET DE SERRE (MIES). - Plan Climat 2004, face au changement climatique, agissons ensemble. - MIES, 2004. - 90 p.

MISSION INTERMINISTÉRIELLE DE L'EFFET DE SERRE (MIES). - Changements climatiques : guide explicatif des accords internationaux. - MIES, 2005. - $60 \mathrm{p}$.

OFFICE NATIONAL DES FORÊTS (ONF). - Forêt et changement climatique : l'essentiel en 20 pages. - Office national des Forêts, 2002. - 20 p.

PIGNARD (G.), DUPOUEY (J.-L.). - Les flux de carbone dans les forêts françaises et européennes : apport des inventaires forestiers. - Comptes rendus de l'Académie d'Agriculture de France, vol. 88, n 5, 2002, pp. 7-17.

RIEDACKER (A.). - Changements climatiques et forêts. - SILVA-RIAT, 2004. - 232 p.

RIEDACKER (A.). - Les forêts et le protocole de Kyoto, le résultat des accords de Bonn et Marrakech en 2001. - La Forêt privée, $n^{\circ}$ 266, juillet-août 2002, pp. 30-37.

SERVICE CENTRAL DES ENQUÊTES ET ÉTUDES STATISTIQUES (SCEES). - L'Utilisation du territoire en 2004. - Agreste Chiffres et Données Agriculture, n 169, août 2005, 65 p.

L'ENTREE EN UIGUEUR DU PROTOCOLE DE KYOTO LE 16 FEURIER 2005 : OUELLES ImPLICATIONS POUA LES FORÊTS FRANÇAISES ? [Résumé]

Le Protocole de Kyoto est entré en vigueur le 16 février 2005 et l'article se propose de faire le point sur le processus en cours et ses conséquences pour les forêts françaises. Il présente successivement la Conventioncadre sur les changements climatiques et le Protocole de Kyoto qui en découle, les textes d'application pour leurs parties concernant spécifiquement le secteur forestier, les modalités d'application pour la forêt française, en particulier les règles comptables, complexes, issues des négociations et enfin les perspectives futures d'un processus qui, non seulement a régulièrement évolué lors de réunions annuelles, mais encore est appelé à poursuivre son développement : le dialogue international sur l'après 2012 a en effet déjà commencé...

\section{THE KYOTO PAOTOCOL BECOMES EFFECTIUE - ImPLICATIONS FOR FRENCH FORESTS [Abstract]}

The Kyoto Protocol came into force on February 16, 2005. The article takes stock of ongoing processes and their consequences for French forests. It first describes the Kyoto Protocol and the framework agreement on climate change from which it is derived, then the particular implementing regulations for forestry stakeholders and the detailed rules for applying it to French forests, and specifically the complex accounting rules that arose from negotiations, and finally the future outlook of these processes that have been regularly revised at annual meetings and will continue to be developed. Indeed, the international dialogue concerning the post-2012 era has already begun. 